Dossier: Mujeres y Educación en la Universidad Nacional

Red de Mujeres Investigadoras de la Universidad Nacional (UNA)

\title{
¿Cómo la robótica educativa impacta a las mujeres estudiantes de secundaria? Un análisis realizado con Python
}

\author{
Irene Hernández Ruiz \\ Universidad Nacional de Costa Rica, Costa Rica \\ irene.hernandez.ruiz@una.cr \\ https://orcid.org/0000-0003-4625-9221 \\ Carolina Gómez Fernández \\ Universidad Nacional de Costa Rica, Costa Rica \\ carolina.gomez.fernandez@una.cr \\ https://orcid.org/0000-0002-1873-8615
}

Richard Arce Vargas Universidad Nacional de Costa Rica, Costa Rica richard.arce.vargas@gmail.com https://orcid.org/0000-0001-9606-2692

Francisco Loría Valverde Universidad Nacional de Costa Rica, Costa Rica franloria06@gmail.com https://orcid.org/0000-0002-3799-2037

Pedro Fonseca Solano Investigador Pensionado, Universidad Nacional de Costa Rica, Costa Rica pfonck2009@gmail.com https://orcid.org/0000-0003-0305-9836

\footnotetext{
(c) (1) (2)

La Revista Estudios es editada por la Universidad de Costa Rica y se distribuye bajo una Licencia Creative Commons Atribución-NoComercial-CompartirIgual 3.0 Costa Rica. Para más información envíe un mensaje a revistaestudios.eeg@ucr.ac.cr.
} 
Dossier: Mujeres y Educación en la Universidad Nacional

Red de Mujeres Investigadoras de la Universidad Nacional (UNA)

Recibido: 4 de agosto de 2020

Aceptado: 10 de noviembre de 2020

Resumen: El siguiente trabajo tiene como objetivo conocer el impacto de la robótica educativa en las estudiantes de secundaria que participaron en el Proyecto Formación de Formadores en Robótica para Colegios en Áreas Vulnerables en Costa Rica, las jóvenes compitieron en el Segundo Encuentro Nacional de Robótica organizado por el proyecto en el año 2019. En esta competencia los participantes pusieron en práctica sus conocimientos de diseño y programación para resolver los desafíos de la actividad. Esta investigación presenta los resultados de un estudio exploratorio al analizar los datos de las mujeres participantes, se aplicó una encuesta física y posteriormente se realizó un análisis utilizando el lenguaje de programación Python, el cual es un gran apoyo en el área de ciencias de datos.

Palabras Clave: género; tecnología; educación; programación en LEGO EV3; Python

\title{
How does educational robotics impact female high school students? An analysis done with Python
}

\begin{abstract}
The following work aims to know the impact of educational robotics on high school students participating in the Project "Training of Trainers in Robotics for Schools in Vulnerable Areas in Costa Rica", the young people competed in the Second National Meeting of Robotics organized for the project in the year 2019. In this competition, the participants put into practice their knowledge of design and programming to solve the challenges of the activity. This research presents the results of an exploratory study when analyzing the data of the participating women, a physical survey was applied and subsequently an analysis was carried out using the Python Programming Language, which is a great support in the area of data science.
\end{abstract}

Keywords: gender; technology; education; LEGO EV3 programming;Python

\section{(C) $(0 \odot$}

La Revista Estudios es editada por la Universidad de Costa Rica y se distribuye bajo una Licencia Creative Commons Atribución-NoComercial-CompartirIgual 3.0 Costa Rica. Para más información envíe un mensaje a 
Dossier: Mujeres y Educación en la Universidad Nacional

Red de Mujeres Investigadoras de la Universidad Nacional (UNA)

\section{Introducción}

Según la Organization for Economic Cooperation and Development (OECD), Costa Rica fue uno de los primeros países de América Latina en lograr una cobertura universal en educación primaria y también en alcanzar que la mayoría de los estudiantes se matriculen en la secundaria. El reto es asegurarse que todos los estudiantes se beneficien de una enseñanza de calidad y de un ambiente de aprendizaje positivo, que completen al menos los 9 años de educación básica y que adquieran sólidas habilidades (OECD, 2017). Existen carreras universitarias en las cuales la participación femenina ha ido en decremento a pesar del auge que se le ha dado a la equidad de género. Por este motivo, Wilson (2002) indica que el problema de la baja participación femenina en el área de la informática se da en dos dimensiones: en el reclutamiento y en la retención, es decir, hay pocas mujeres que se matriculan en las carreras de informática y una vez matriculadas es difícil mantenerlas empadronadas.

En el año 2012, en la Universidad Nacional de Costa Rica, como lo indicaron Mata, Quesada y Raventós (2012) no se mostró una tendencia a la baja en el comportamiento de ingreso de las mujeres a la carrera Ingeniería en Sistemas de Información.

Los datos de ingreso suministrados por el Departamento de Registro de la Universidad Nacional (2020) indican que en el I Ciclo del año 2020 a la Carrera de Ingeniería de Sistemas de Información ingresaron 249 estudiantes, de los cuales 195 son hombres $(78,31 \%)$ y 54 son mujeres $(21,68 \%)$, de esta manera queda en

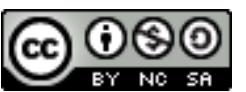

La Revista Estudios es editada por la Universidad de Costa Rica y se distribuye bajo una Licencia Creative Commons Atribución-NoComercial-CompartirIgual 3.0 Costa Rica. Para más información envíe un mensaje a 
Dossier: Mujeres y Educación en la Universidad Nacional

Red de Mujeres Investigadoras de la Universidad Nacional (UNA)

evidencia que el número de estudiantes de género femenino es menos de un cuarto del total de ingreso.

El estudio de Mata et al (2012), demostró que existe una considerable brecha de género en los programas de ciencias de la computación en las Universidades Públicas de Costa Rica, considerando el porcentaje de mujeres graduadas en el período 2001-2009, el cual muestra una notable disminución de casi un punto porcentual anual especialmente después del 2006.

En Costa Rica organizaciones y empresas han empezado a crear proyectos para impulsar la participación y liderazgo del género femenino en el área de la tecnología, un ejemplo es Sulá Batsú (2017) esta empresa posee el proyecto llamado "Café Tecnológico", que se basa en crear diferentes reuniones en donde las mujeres discuten sobre innovación, nuevas metodologías y tendencias tecnológicas. Además, las participantes pueden compartir casos reales sobre ser mujer en el área tecnológica. Durante la actividad, se realizan diferentes talleres como enseñar lenguajes de programación como Python o metodologías como Design Thinking.

Es importante incentivar a las mujeres desde pequeñas a seguir un camino en el área de la tecnología, como el proyecto realizado por el Instituto Tecnológico de Estudios Superiores de Monterrey de México (ITESM) y el Massachusetts Institute of Technology (MIT) de Estados Unidos citado por Mariano (2018), en donde estudiantes del MIT impartían el curso llamado "Patrones Hermosos" a mujeres estudiantes de secundaria entre 13 y 17 años, en donde se buscaba motivarlas a estudiar carreras en informática. Sin embargo, la importancia de estos talleres es que las jóvenes interesadas tengan un espacio para aprender y de esta manera fomentar más la participación femenina.

\section{(c) (i) (2)}

La Revista Estudios es editada por la Universidad de Costa Rica y se distribuye bajo una Licencia Creative Commons Atribución-NoComercial-CompartirIgual 3.0 Costa Rica. Para más información envíe un mensaje a 
Dossier: Mujeres y Educación en la Universidad Nacional

Red de Mujeres Investigadoras de la Universidad Nacional (UNA)

En Costa Rica también existen estas prácticas para motivar a niñas y jóvenes. En el Instituto Tecnológico de Costa Rica (TEC) se creó una comisión de género y tecnología. En conjunto con estudiantes y docentes se realizan dinámicas grupales para promover equidad de género y la ciencia, además de atraer mujeres hacia las ciencias y la formación técnica en secundaria y primaria (Jiménez, 2019). Este tipo de actividades son de suma importancia para eliminar los diferentes estereotipos existentes sobre la mujer y su participación en carreras tecnológicas e incorporarlas en las áreas de ciencia y tecnología, en donde pueden destacar y contribuir a la sociedad sin tener miedo a la represión social.

Las razones expuestas anteriormente fundamentan la importancia del fomento de las tecnologías, por esto, uno de los objetivos del proyecto Proyecto Formación de Formadores en Robótica para Colegios en Áreas Vulnerables de Costa Rica, ha sido la incorporación de mujeres estudiantes de colegios públicos en el área de la robótica educativa. En el año 2019, se llevó a cabo la actividad del Segundo Encuentro Nacional de Robótica con la participación de 35 colegios beneficiarios del proyecto. En esta actividad los estudiantes participaron realizando retos de robótica los cuales fueron diseñados por el equipo organizador del torneo. Se consideró importante, realizar una valoración del evento por parte de los estudiantes y para ello se realizó una encuesta física que permitiera recopilar la opinión de todos los estudiantes. Para el análisis de la información fue importante considerar herramientas científicas que permitieran procesar determinado volumen en pocos pasos, por este motivo se seleccionó el programa Python.

\footnotetext{
c) (i)(2)

La Revista Estudios es editada por la Universidad de Costa Rica y se distribuye bajo una Licencia Creative Commons Atribución-NoComercial-CompartirIgual 3.0 Costa Rica. Para más información envíe un mensaje a revistaestudios.eeg@ucr.ac.cr.
} 
Dossier: Mujeres y Educación en la Universidad Nacional

Red de Mujeres Investigadoras de la Universidad Nacional (UNA)

\section{Python para el análisis de datos}

Un lenguaje de programación como lo indica Olarte: "es un lenguaje formal diseñado para realizar procesos que pueden ser llevados a cabo por máquinas como las computadoras" (2018). Existe una diversidad de lenguajes, por ejemplo, están los lenguajes de máquina (bajo nivel), el lenguaje ensamblador y el lenguaje de alto nivel como lo es Python donde se permite escribir códigos utilizando instrucciones, que luego de ser traducidos la computadora puede ejecutar.

Como lo indican Challenger-Pérez, Díaz-Ricardo y Becerra-García "Python fue creado por Guido van Rossum, un programador holandés a finales de los 80 y principio de los 90 cuando se encontraba trabajando en el sistema operativo Amoeba" (2014, p.3), las versiones fueron evolucionando hasta llegar actualmente hasta Python 3.x Rochina (2016) indica que Python es un programa óptimo para el análisis de datos debido a los siguientes aspectos:

1. Las numerosas librerías creadas para esta finalidad como:

a) Numpy y Pandas que implementan funciones para cálculos matemáticos y estadísticos

b) Mlpy con algoritmos de aprendizaje máquina

c) Matploit que permite la visualización y representación gráfica de los datos.

2. Su integración con aplicaciones como MongoDB (base de datos no relacional), Hadoop o Pentaho.

\section{(c) (i)(2)}

La Revista Estudios es editada por la Universidad de Costa Rica y se distribuye bajo una Licencia Creative Commons Atribución-NoComercial-CompartirIgual 3.0 Costa Rica. Para más información envíe un mensaje a 
Dossier: Mujeres y Educación en la Universidad Nacional

Red de Mujeres Investigadoras de la Universidad Nacional (UNA)

Si a todo ello se le suma la fácil y rápida curva de aprendizaje junto con su versatilidad, es Python entonces un lenguaje de gran calidad para los analistas de datos. Por esos motivos el procesamiento de los datos se hizo utilizando Python, además se espera que sea una motivación para futuros investigadores hacer uso de esta herramienta.

\section{Proyecto Formación de Formadores en robótica para colegios en áreas vulnerables de Costa Rica}

Desde el año 2015 se creó el proyecto denominado Formación de Formadores en Robótica para Colegios en Áreas Vulnerables de Costa Rica, iniciativa conjunta de la Escuela de Informática de la Universidad Nacional, el Instituto Costarricense sobre Drogas (ICD) y el Ministerio de Educación Pública de Costa Rica (MEP). proyecto se enfocaba en la capacitación de profesores en el área de robótica educativa, quienes posteriormente realizaban talleres con estudiantes de secundaria con el fin de generar espacios para que estos ocuparan su tiempo libre en actividades positivas haciendo uso de la robótica educativa (Fonseca y Hernández, 2017). Como parte del seguimiento a los docentes y a los estudiantes, el 27 de septiembre del 2019 se desarrolló el Encuentro Nacional de Robótica (ver anexo 2), en el cual participaron 200 estudiantes de 35 colegios pertenecientes al proyecto y se realizaron retos en 5 categorías. En este evento, se evidenció el conocimiento que han adquirido los jóvenes en el área de la programación en un entorno gráfico

\section{(C) $(0 \odot$}

La Revista Estudios es editada por la Universidad de Costa Rica y se distribuye bajo una Licencia Creative Commons Atribución-NoComercial-CompartirIgual 3.0 Costa Rica. Para más información envíe un mensaje a 
Dossier: Mujeres y Educación en la Universidad Nacional

Red de Mujeres Investigadoras de la Universidad Nacional (UNA)

\section{Metodología aplicada}

Este trabajo es un estudio exploratorio, que permite conocer la percepción de las mujeres estudiantes de colegios beneficiarios del proyecto acerca de su aprendizaje sobre la robótica educativa. Se utilizó como método de trabajo una encuesta física, fue completada por 186 estudiantes de los 200 participantes. De las respuestas 42 son mujeres $(22,5 \%)$ y 144 hombres $(77,5 \%)$. El instrumento utilizado se presenta en la Figura 1, se trabajó principalmente información sobre las emociones presentadas por parte del estudiantado.

- ¿Entre las emociones y sentimientos vividos a través de la robótica experimente los siguientes: ? Puede marcar varias opciones:

() Motivación () Satisfacción () Realización ( ) Felicidad ( ) Frustración () Otra, cuál:

- Comente cómo ha sido el apoyo de las profesoras y profesores en los talleres:

- Durante los talleres de robótica he logrado una mejor: Puede marcar varias opciones ( ) Toma de decisiones asertivas ( ) Manejo del estrés ( ) Comunicación asertiva () Autoestima () Resolución de problemas () Conocimiento de sí mismo () Presión de grupo ( ) Manejo de las emociones ( ) Habilidad de pensar en forma crítica ( ) Convivencia ( ) Trabajo en equipo

- ¿Le gustaría seguir con los talleres de robótica?( ) Si ( ) No ( ) Por cuál razón:

- ¿Le gustaría participar más en este tipo de eventos? ( ) Si ( ) No ( ) Por cual razón

- ¿Le gustaría conocer la Escuela de informática de la Universidad Nacional de Costa Rica? () Si ( ) No ( ) En otro momento

- Algún comentario adicional que quisiera brindar:

Figura 1. Cuestionario utilizado.

Fuente elaboración propia

La Revista Estudios es editada por la Universidad de Costa Rica y se distribuye bajo una Licencia Creative Commons Atribución-NoComercial-CompartirIgual 3.0 Costa Rica. Para más información envíe un mensaje a revistaestudios.eeg@ucr.ac.cr. 
Dossier: Mujeres y Educación en la Universidad Nacional

Red de Mujeres Investigadoras de la Universidad Nacional (UNA)

Pasos para la recolección de la información y su procesamiento:

1. Tabulación de los 186 cuestionarios. La información se colocó en una tabla de una hoja de cálculo.

2. Posteriormente, se tomaron algunos cuestionarios al azar para garantizar que la información no tuviese errores de digitación.

3. Seguidamente se ordenó la información en la hoja de cálculo y se separó la información de las mujeres estudiantes ya que se requiere resaltar el trabajo por parte de ellas.

4. Se incorporó el uso de las librerías necesarias en Python para poder hacer el gráfico según las emociones percibidas.

5. Se estableció la apertura del archivo de Excel con la información y se procedió a colocar las instrucciones necesarias para el procesamiento de la información, las cuales son:

a) Hacer un conjunto de datos denominado dataset. Esta estructura tiene como funciones la de contar para obtener frecuencias y colocar las categorías de esas frecuencias. En este caso se determinó como esos elementos: motivación, satisfacción, realización, felicidad y frustración.Posteriormente haciendo uso de instrucciones como count permiten contar las categorías y las frecuencias y luego listarlas según el grupo escogido.

6. La graficación se realizó con la instrucción countplot para obtener las frecuencias de las respuestas de las estudiantes. Como puede observarse en la

\section{(c) (i) (9) (2)}

La Revista Estudios es editada por la Universidad de Costa Rica y se distribuye bajo una Licencia Creative Commons Atribución-NoComercial-Compartirlgual 3.0 Costa Rica. Para más información envíe un mensaje a 
Dossier: Mujeres y Educación en la Universidad Nacional

Red de Mujeres Investigadoras de la Universidad Nacional (UNA)

Figura 2, el procesamiento de la información requiere muy pocos pasos y permite generar los gráficos logrando que los volúmenes de información sean pequeños.

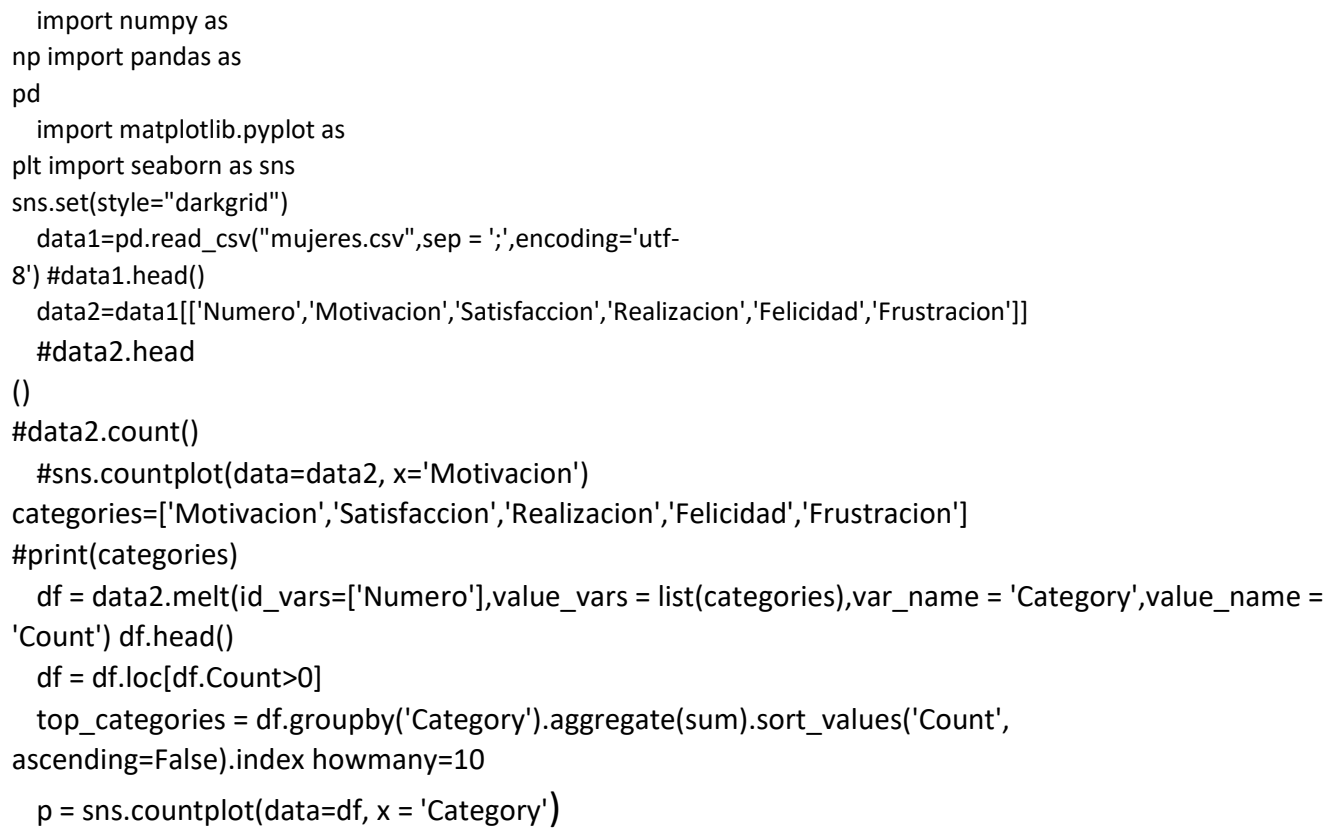

Figura 2. Código necesario para la generación de gráficos en Python.

Fuente: Elaboración Propia.

7. En el tema de las habilidades desarrolladas, si se consideró importante presentar la opinión de los estudiantes tanto de las mujeres como de los hombres estudiantes.

\section{(C) $(0 \odot$}

La Revista Estudios es editada por la Universidad de Costa Rica y se distribuye bajo una Licencia Creative Commons Atribución-NoComercial-CompartirIgual 3.0 Costa Rica. Para más información envíe un mensaje a revistaestudios.eeg@ucr.ac.cr. 
Dossier: Mujeres y Educación en la Universidad Nacional

Red de Mujeres Investigadoras de la Universidad Nacional (UNA)

\section{Principales resultados}

1. Análisis de sentimientos experimentados por los estudiantes:

En el Gráfico 1 se presentan las frecuencias de las emociones que registraron las estudiantes. Se encontró una gran motivación y felicidad. Esto significó que la olimpiada originó que los estudiantes salieran de la rutina diaria de lecciones y lograran compartir con sus pares de otros colegios.

Como puede verse en el Gráfico 1, la realización fue la que las estudiantes marcaron menos veces con 23 ocurrencias. Esto quiere decir que no todos los estudiantes experimentaron ese sentimiento durante la olimpiada.

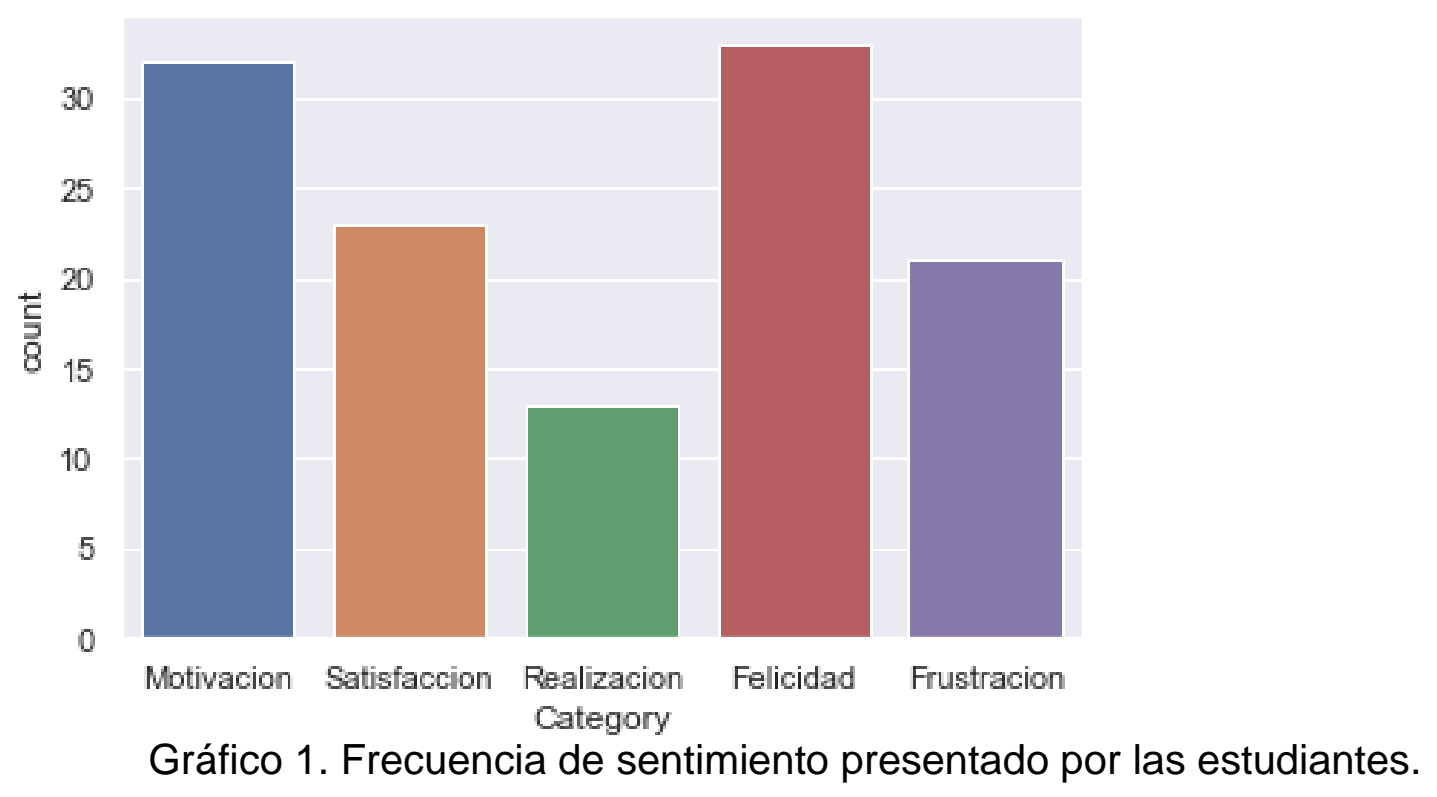

Fuente: Elaboración Propia.

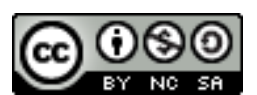

La Revista Estudios es editada por la Universidad de Costa Rica y se distribuye bajo una Licencia Creative Commons Atribución-NoComercial-CompartirIgual 3.0 Costa Rica. Para más información envíe un mensaje a revistaestudios.eeg@ucr.ac.cr. 
Dossier: Mujeres y Educación en la Universidad Nacional

Red de Mujeres Investigadoras de la Universidad Nacional (UNA)

Con respecto a las razones por las cuales les gustaría continuar participando en los talleres, en la Tabla 1, se registran las respuestas indicadas por las estudiantes, con el fin de valorar su percepción en continuar con este tipo de talleres.

\begin{tabular}{|c|c|}
\hline \multicolumn{2}{|c|}{ Razones por las cuales les gustaría continuar con los talleres: } \\
\hline Aprender más & Para aprender más \\
\hline Es divertido y emocionante & Para disfrutar y vacilar \\
\hline $\begin{array}{l}\text { Es divertido, pero no me } \\
\text { apasiona }\end{array}$ & Por diversión \\
\hline Es muy interesante & Por lo entretenido y productivo \\
\hline Es una actividad muy interesante & Por motivación \\
\hline La robótica en sí es un buen taller & Porque aprendo más \\
\hline Me atrae mucho & Porque es divertido \\
\hline $\begin{array}{c}\text { Me ayuda a concentrarme más y } \\
\text { desestresarme }\end{array}$ & $\begin{array}{l}\text { Porque es interesante aprender } \\
\text { cosas } \\
\text { nuevas }\end{array}$ \\
\hline Me desestresan y me encantan & Porque es muy interesante \\
\hline Me gusta & $\begin{array}{l}\text { Porque es un "plus" en mi } \\
\text { especialidad (Electrónica Industrial) } \\
\text { para la parte de } \\
\text { Sistemas de Información }\end{array}$ \\
\hline $\begin{array}{l}\text { Me gusta mucho trabajar con } \\
\text { robots }\end{array}$ & $\begin{array}{l}\text { Porque es un gran medio de } \\
\text { distracción }\end{array}$ \\
\hline $\begin{array}{l}\text { Porque gracias a ello conocí mis } \\
\text { conocimientos }\end{array}$ & $\begin{array}{l}\text { Porque me parece un taller muy } \\
\text { creativo }\end{array}$ \\
\hline $\begin{array}{c}\text { Porque me apasiona y me } \\
\text { gustaría }\end{array}$ & $\begin{array}{l}\text { Porque quiero aprender a hacer un } \\
\text { robot }\end{array}$ \\
\hline
\end{tabular}

La Revista Estudios es editada por la Universidad de Costa Rica y se distribuye bajo una Licencia Creative Commons Atribución-NoComercial-CompartirIgual 3.0 Costa Rica. Para más información envíe un mensaje a revistaestudios.eeg@ucr.ac.cr. 
Dossier: Mujeres y Educación en la Universidad Nacional

Red de Mujeres Investigadoras de la Universidad Nacional (UNA)

\begin{tabular}{|c|c|}
\hline seguir aprendiendo & \\
\hline $\begin{array}{c}\text { Porque me ayuda mucho a } \\
\text { distraerme }\end{array}$ & Porque siento que tengo capacidad \\
\hline Porque me encanta la robótica & Porque son bonitos \\
\hline $\begin{array}{c}\text { Porque me encanta, es una linda } \\
\text { experiencia }\end{array}$ & $\begin{array}{c}\text { Porque son demasiado chivas y } \\
\text { divertidos }\end{array}$ \\
\hline $\begin{array}{c}\text { Son muy divertidos. Conozco } \\
\text { personas }\end{array}$ & Porque son muy atractivos \\
\hline $\begin{array}{c}\text { Tener un pasatiempo mejor } \\
\text { entretiene }\end{array}$ \\
\hline $\begin{array}{c}\text { Ya que me siento a gusto } \\
\text { armando y } \\
\text { programando }\end{array}$ & Porque uno se motiva y distracción \\
\hline Son divertidos & Son muy buenos \\
\hline
\end{tabular}

Tabla 1. Razones por las cuales les gustaría continuar en el taller

Como puede visualizarse en la Tabla 1, las estudiantes mujeres se sienten muy cómodas en este tipo de talleres, a su vez, les gusta la robótica educativa porque la visualizan como una actividad que les ayuda a distraerse.

Por otra parte en la Tabla 2, se analizaron las respuestas tanto de los hombres y mujeres estudiantes, se les preguntó a cada uno acerca de las habilidades que han desarrollado hasta el momento con la robótica educativa en los talleres, dentro de

\section{() $(\Theta \odot$}

La Revista Estudios es editada por la Universidad de Costa Rica y se distribuye bajo una Licencia Creative Commons Atribución-NoComercial-CompartirIgual 3.0 Costa Rica. Para más información envíe un mensaje a revistaestudios.eeg@ucr.ac.cr. 
Dossier: Mujeres y Educación en la Universidad Nacional

Red de Mujeres Investigadoras de la Universidad Nacional (UNA)

las respuestas se destacó el trabajo en equipo (109 respuestas), en segundo lugar, el del manejo del estrés y en último lugar el manejo de la autoestima.

\begin{tabular}{|l|c|}
\hline Toma de decisiones asertivas & 69 \\
\hline Comunicación asertiva & 48 \\
\hline Resolución de problemas & 88 \\
\hline Presión de grupo & 63 \\
\hline Habilidad de pensar en forma crítica & 70 \\
\hline Trabajo en equipo & 109 \\
\hline Manejo del estrés & 91 \\
\hline Autoestima & 38 \\
\hline Conocimiento de sí mismo & 53 \\
\hline Manejo de las emociones & 65 \\
\hline Convivencia & 80 \\
\hline
\end{tabular}

Tabla 2. Razones por las cuales les gustaría continuar en el taller

\section{Conclusiones}

Actividades como las olimpiadas de robótica generan una gran motivación en las estudiantes, son experiencias de vida, debido a que logran tener un convivio con sus compañeros y con integrantes de otros colegios. Aplicar sus ideas para participar en un equipo de trabajo les permite interactuar con las implicaciones que conllevan el contacto entre las personas. El trabajo realizado y su enfoque evidencian que este tipo de eventos motivan a todos los participantes, por lo cual es de suma importancia seguir organizando olimpiadas para que otras generaciones de estudiantes puedan aprovechar estas oportunidades.

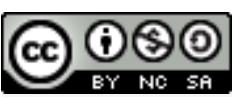

La Revista Estudios es editada por la Universidad de Costa Rica y se distribuye bajo una Licencia Creative Commons Atribución-NoComercial-CompartirIgual 3.0 Costa Rica. Para más información envíe un mensaje a revistaestudios.eeg@ucr.ac.cr. 
Dossier: Mujeres y Educación en la Universidad Nacional

Red de Mujeres Investigadoras de la Universidad Nacional (UNA)

Para contribuir a la participación de las niñas en las ciencias y tecnologías, se requieren espacios de convivencia para que ellas se motiven, visualicen y valoren la participación de sus profesoras como modelos de inspiración a la hora de pensar en una posible carrera universitaria en esta línea. Por otra parte, queda en evidencia que los lenguajes de programación promueven que los análisis de datos sean una tendencia hoy en día para la toma de decisiones y para conocer mejor a la población. Por estas razones es que se promueve el uso del lenguaje de programación Python, el cual es muy útil por lo que permite generar resúmenes con información en poco tiempo y con una gran calidad de los gráficos. Y esto permite a investigadores acceder de una manera sistemática a sus datos y aumentar la producción científica a nivel nacional.

\section{Anexo 1}

En la siguiente lista se encuentran algunos enlaces de diferentes medios de comunicación de Costa Rica que le dieron cobertura al Encuentro Nacional de Robótica

- $\quad$ https://www.mep.go.cr/noticias/estudiantes-disfrutan-robotica-comoestrategia-prevencion-contra-drogas

- $\quad$ https://teletica.com/236627_proyecto-utiliza-la-robotica-para-alejar-aestudiantes-de-las-drogas

- $\quad$ https://diarioextra.com/Noticia/detalle/399546/se-alejan-de-drogas-conrobotica

\section{(c) (i) (2) (2)}

La Revista Estudios es editada por la Universidad de Costa Rica y se distribuye bajo una Licencia Creative Commons Atribución-NoComercial-CompartirIgual 3.0 Costa Rica. Para más información envíe un mensaje a revistaestudios.eeg@ucr.ac.cr. 
Dossier: Mujeres y Educación en la Universidad Nacional

Red de Mujeres Investigadoras de la Universidad Nacional (UNA)

- $\quad$ http://www.laprensalibre.cr/Noticias/detalle/155504/200-estudiantesaprenden-robotica-y-le-dicen-no-a-drogas

- $\quad h t t p s: / / w w w . y o u t u b e . c o m / w a t c h ? v=F d A G 1 i V K l p Q \& f e a t u r e=y o u t u . b e \& l i s t=U$ UG_15BGn70uq9rpveZkO0WQ

- $\quad$ https://www.crhoy.com/nacionales/mep-combate-las-drogas-conprogramas-de-robotica/

\section{Anexo 2}

Enlace del Encuentro de Robótica: https://pfonsecalice.jimdofree.com/

\section{Referencias bibliográficas}

Challenger-Pérez, I., Díaz-Ricardo, y., Becerra-García, R. (2014). El lenguaje de programación Python. Ciencia Holguín. 20(2). 1-13. Obtenido de https://www.redalyc.org/pdf/1815/181531232001.pdf el 6 de junio de 2020

Departamento de Registro de la Universidad Nacional (2020). Estudiantes que ingresaron a la Escuela de Informática en el año 2020. Comunicación personal

Fonseca, P. y Hernández, I. (2017) Training of robotic trainers for schools in vulnerable areas of Costa Rica: Use of ICT to help Costa Rican youth avoid drug use, 2017 XLIII Latin American Computer Conference (CLEI), Cordoba, 2017, 1-7, doi: 10.1109/CLEI.2017.8226421.

\section{(C) $(\Theta \odot$}

La Revista Estudios es editada por la Universidad de Costa Rica y se distribuye bajo una Licencia Creative Commons Atribución-NoComercial-CompartirIgual 3.0 Costa Rica. Para más información envíe un mensaje a revistaestudios.eeg@ucr.ac.cr. 
Dossier: Mujeres y Educación en la Universidad Nacional

Red de Mujeres Investigadoras de la Universidad Nacional (UNA)

Jiménez, G. (2019). Combatir estereotipos, clave para aumentar presencia femenina en carreras tecnológicas. Hoy en el TEC. Obtenido de de: https://www.tec.ac.cr/hoyeneltec/2019/03/12/combatir-estereotipos-clave-

aumentar-presencia-femenina-carreras-tecnologicas el 17 de enero de 2020

Mariano, M. (2018). En alianza con MIT acercan a niñas a la informática.[falta el contenedor del artículo 0 de la información] Obtenido de https://tec.mx/es/noticias/monterrey/educacion/en-alianza-con-mit-acercan-ninasla-informatica el 14 de enero de 2020

Mata, F. J., Quesada, A. y Raventós, G. M. (2012). Gender gap in computer science programs from Costa Rican Public universities. XXXVIII Conferencia Latinoamericana En Informatica (CLEI). Medellín, Colombia. DOI: 10.1109/CLEl.2012.6427245

OECD (2017). Educación en Costa Rica. Aspectos destacados 2017. Obtenido de http://www.rectoria.ucr.ac.cr/site/wp-content/uploads/2017/08/aspectosdestacados-2017.pdf el 7 de mayo de 2020.

Olarte Gervacio, L. (2018) Lenguaje de Programación. Conogasi, Conocimiento para la vida. Fecha de consulta: Octubre 12, 2020

Rochina, P. (2016). Python vs R para el análisis de datos. Obtenido de https://revistadigital.inesem.es/informatica-y-tics/python-r-analisis-datos/ el 6 de junio de 2020. Revista digital INESEM.

Sulá Batsú (2017). "Café Tecnológico" celebrará el liderazgo femenino en las Tecnologías Digitales. Obtenido de 
ISSN 1659-3316

Dossier: Mujeres y Educación en la Universidad Nacional

Red de Mujeres Investigadoras de la Universidad Nacional (UNA)

https://www.sulabatsu.com/blog/noticias/cafe-tecnologico-celebrara-el- liderazgofemenino-en-las-tecnologias-digitales/ el 14 de enero de 2020

Wilson, B. C. (2002). A study of factors promoting success in computer science including gender differences. Educación en Ciencias de la Computación, 12(1-2), 141-164. doi: 10.1076/csed.12.1.141.8211 